\title{
Green tea protects against hippocampal neuronal apoptosis in diabetic encephalopathy by inhibiting JNK/MLCK signaling
}

\author{
YONGJIE XU ${ }^{1,2}$, SHENGJU LIU ${ }^{1}$, LIYING ZHU ${ }^{1}$, LONGGUANG DAI ${ }^{1}$, WEN QIAN ${ }^{1}$, \\ JINGZHI ZHANG ${ }^{1},{\text { XING } \mathrm{LI}^{3} \text { and WEI PAN }}^{1,2}$ \\ ${ }^{1}$ Department of Medical Laboratory, Affiliated Hospital of Guizhou Medical University, Guiyang Medical College; \\ ${ }^{2}$ School of Public Health, Key Laboratory of Environmental Pollution Monitoring and Disease Control, \\ Guizhou Medical University; ${ }^{3}$ Guizhou University of Traditional Chinese Medicine, \\ Guiyang, Guizhou 550004, P.R. China
}

Received October 31, 2020; Accepted April 15, 2021

DOI: $10.3892 / \mathrm{mmr} .2021 .12214$

\begin{abstract}
Although diabetic encephalopathy (DE) is a major late complication of diabetes, the pathophysiology of postural instability in DE remains poorly understood. Prior studies have suggested that neuronal apoptosis is closely associated with cognitive function, but the mechanism remains to be elucidated. Green tea, which is a non-fermented tea, contains a number of tea polyphenols, alkaloids, amino acids, polysaccharides and other components. Some studies have found that drinking green tea can reduce the incidence of neurodegenerative diseases and improve cognitive dysfunction. We previously found that myosin light chain kinase (MLCK) regulates apoptosis in high glucose-induced hippocampal neurons. In neurodegenerative diseases, including Alzheimer's disease and Parkinson's disease, activation of the JNK signaling pathway promotes neuronal apoptosis. However, the relationship between JNK and MLCK remains to be elucidated. Green tea serum was obtained using seropharmacological methods and applied to hippocampal neurons. In addition, a type 1 diabetes rat model was established and green tea extract was administered, and the Morris water maze test, Cell Counting Kit- 8 assays, flow cytometry, western blotting and terminal deoxynucleotidyl transferase-mediated dUTP nick end-labelling assays were used to examine the effects of green tea on hippocampal neuronal apoptosis in diabetic rats. The results
\end{abstract}

Correspondence to: Dr Xing Li, Guizhou University of Traditional Chinese Medicine, 1 Dongqing South Road, Gui'an, Guiyang, Guizhou 550004, P.R. China

E-mail: lixing_1211@sina.com

Dr Wei Pan, Department of Medical Laboratory, Affiliated Hospital of Guizhou Medical University, Guiyang Medical College, 9 Beijing Road, Guiyang, Guizhou 550004, P.R. China

E-mail:pw_6602@sina.com

Key words: diabetic encephalopathy, hippocampal neuron apoptosis, green tea, JNK/myosin light chain kinase signaling pathway, cognitive function demonstrated that green tea can protect against hippocampal neuronal apoptosis by inhibiting the JNK/MLCK pathway and ultimately improves cognitive function in diabetic rats. The present study provided novel insights into the neuroprotective effects of green tea.

\section{Introduction}

Diabetic encephalopathy (DE) is a serious chronic complication of diabetes $(1,2)$ and its clinical manifestations include loss of learning and memory abilities, and cognitive dysfunction (3). At present, the pathogenesis of DE is unclear, but substantial data have shown that neuronal apoptosis is one of the key pathogenic mechanisms of DE $(2,4)$. Studies have found that neurons are regulated by the mitochondrial apoptosis pathway, death receptor apoptosis pathway and endoplasmic reticulum apoptosis pathway and these pathways ultimately affect cognitive function $(4,5)$. A recent study revealed that Ganoderma lucidum triterpenoids improve cognitive impairment, alleviate neuronal damage and inhibit apoptosis in hippocampal tissues and cells in Alzheimer's disease by inhibiting the Rho-associated protein kinase 1 signaling pathway (6). Although research has shown that the hippocampus is the main lesion site in DE and that hippocampal neuronal apoptosis is closely associated with the occurrence of cognitive dysfunction, the mechanism of action remains to be elucidated (7).

Myosin light chain kinase (MLCK), a key molecule in the calcium signaling pathway (8), is expressed in hippocampal neurons, where it can accelerate the mobilization of synaptic vesicles, promote the release of various neurotransmitters and regulate the elongation of growth cones $(9,10)$. A previous study found that the increase in nerve cell apoptosis observed in a rat model of ischemia-reperfusion brain injury might be associated with upregulation of MLCK expression and that the addition of a specific MLCK inhibitor (ML-7) significantly reduces neuronal apoptosis (11). In addition, increased MLCK expression affects the cytoskeletal structure and this effect leads to the occurrence of apoptosis (12). Our previous studies found that MLCK serves a role in hippocampal neuronal apoptosis, but the regulatory mechanism 
needs further examination $(13,14)$. Previous research has shown that hippocampal neuronal apoptosis is regulated by multiple pathways, including the MAPK/JNK signaling pathway and the Wnt/ $\beta$-catenin signaling pathway (15). The JNK signaling pathway not only serves an important role in regulating apoptosis, but is also associated with neurodegenerative diseases, such as Alzheimer's disease (16). A number of factors, including inflammatory cytokines, oxidative damage and a high-glucose (HG) environment, can activate the JNK signaling pathway (17) and thereby the mitochondrial apoptotic pathway, which affects expression of the apoptosis-related proteins Bcl-2 and Bax, induces the release of cytochrome c from mitochondria and activates the downstream caspase signaling pathway (18). However, JNK does not act universally as a pro-apoptotic signaling pathway because JNK activation can also exert antiapoptotic activity in certain situations (19). As the role of the JNK signaling pathway in DE has not been fully elucidated, the present study aimed to clarify the relationship between the JNK signaling pathway and MLCK in the apoptosis of hippocampal neurons in DE.

Green tea, a non-fermented tea, contains a number of tea polyphenols, alkaloids, amino acids, polysaccharides and other components. A typical brewed green tea contains $30-45 \%$ green tea catechins, including epicatechin (EC), epicatechin-3-gallate (ECG), epigallocatechin and epigallocatechin-3-gallate (EGCG) (20). A previous study suggested that green tea has anti-diabetic and anti-ageing activity and lowers the blood cholesterol leve (20), which results in reduced incidence of neurodegenerative diseases and protects the nervous system (21). Epidemiological research has begun to show that green tea suppresses cognitive decline (22).

In diabetes development, whether green tea can protect against the cognitive dysfunction caused by diabetes and the potential pharmacological mechanism remains to be elucidated. Therefore, the purpose of the present study was to elucidate the mechanism of action of green tea in hippocampal neuronal apoptosis in DE. It is hoped that the present study will contribute to a deeper understanding of the pharmacological mechanism of green tea.

\section{Materials and methods}

Experimental animals. A total of 300 healthy newborn (24-h old) Sprague-Dawley (SD) rats (6-12 g) and 66 healthy adult SD rats (180-220 g, male) were purchased from the Experimental Animal Center of Guizhou Medical University [animal license no. SYXK(Qian)2018-0001, Guiyang, China]. The 300 newborn SD rats were used to cultivate primary hippocampal neurons; generally, 10 newborn rats provided $2 \times 10^{5}$ hippocampal neurons and primary hippocampal neurons do not divide in culture. In addition, 60 adult SD rats were used to establish a type 1 diabetes rat model and six adult rats were used to obtain serum. Prior to the experiments, the animals were maintained under a 12-h light/dark cycle in an indoor environment with a temperature of $22 \pm 2^{\circ} \mathrm{C}$ and $50 \pm 10 \%$ humidity, and provided with sterile water and food ad libitum. The experiments were performed in strict accordance with the guidelines established by the National Institutes of Health for the Use of Laboratory Animals (National Institutes of Health publication no. 85-23, 1985) and the experiments were approved by the Guizhou Medical University Animal Care and Use Committee (approval no. 1800954; Guiyang, China).

Preparation of green tea serum and blank serum. Blank serum and green tea serum were prepared according to a previous study (23). Green tea powder (20.0 g) was weighed and $100 \mathrm{ml}$ sterile distilled water was added to the powder. The solution was mixed in a water bath shaker at $70^{\circ} \mathrm{C}$ for $1 \mathrm{~h}$. The mixture was filtered through a sieve and green tea extract was then obtained for intragastric use. A total of six healthy SD rats were randomly selected and divided into one of two groups: The rats in the control group were intragastrically administered normal saline at a dosage of $20 \mathrm{ml} / \mathrm{kg} /$ day and those in the model group were intragastrically administered green tea concentrate at a dosage of $20 \mathrm{ml} / \mathrm{kg}$ twice per day for 3 consecutive days. After $1.5 \mathrm{~h}$ of gavage on the last day of treatment, the SD rats were anaesthetized via intraperitoneal injection of sodium pentobarbital $(30 \mathrm{mg} / \mathrm{kg})$ until they lost consciousness. Subsequently, $5 \mathrm{ml}$ of blood was drawn from the femoral artery (peripheral blood) and centrifuged at $1,006.2 \mathrm{x} \mathrm{g}$ for $10 \mathrm{~min}$ at $4{ }^{\circ} \mathrm{C}$ and the upper layer containing serum was aspirated. The rats were sacrificed via cervical dislocation. The green tea serum and blank serum were then inactivated at $56^{\circ} \mathrm{C}$ for $30 \mathrm{~min}$ and diluted in maintenance medium to obtain low-dose blank serum (25\% blank serum), medium-dose blank serum (50\% blank serum), high-dose blank serum (100\% blank serum), low-dose green tea serum (25\% green tea serum), medium-dose green tea serum (50\% green tea serum) and high-dose green tea serum (100\% green tea serum). All sera were stored in an ultra-low temperature refrigerator at $-80^{\circ} \mathrm{C}$.

High-performance liquid chromatography (HPLC). The components in the blank serum and green tea serum were detected by HPLC using an Agilent 1100 system (Agilent Technologies, Inc.) according to a previous study (24). First, the serum samples were pretreated and the HPLC test conditions confirmed: Diamonsil C18 column $(250 \times 4.6 \mathrm{~mm}$, $5 \mu \mathrm{m}$ ), mobile phase consisting of acetonitrile (A) and $0.03 \%$ phosphoric acid aqueous solution (B), gradient elution (0 min, $8 \%$ A; $18 \mathrm{~min}, 19 \%$ A; $25 \mathrm{~min}, 23 \% \mathrm{~A} ; 45 \mathrm{~min}, 26 \% \mathrm{~A}$; $70 \mathrm{~min}, 37 \% \mathrm{~A}$; $80 \mathrm{~min}, 63 \% \mathrm{~A}$; and $95 \mathrm{~min}, 70 \% \mathrm{~A})$, volume flow rate of $0.9 \mathrm{ml} / \mathrm{min}$, column temperature of $30^{\circ} \mathrm{C}$, detection wavelength of $278 \mathrm{~nm}$ and injection volume of $10 \mu 1$.

Hippocampal neuronal cell culture and identification. The primary hippocampal neurons were cultured according to a previously published method (14). Newborn SD rat were sacrificed by cervical dislocation and the mortality of the animals confirmed by cessation of heartbeat. Primary hippocampal neurons were extracted from newborn rat hippocampal tissue. The basal medium glucose concentration was $25 \mathrm{mmol} / \mathrm{l}$ glucose (Neurobasal-A medium, without FBS; Gibco; Thermo Fisher Scientific, Inc.). After 7 days of culture, the hippocampal neurons were incubated with anti-neuron specific enolase (NSE) antibody (1:100; cat. no. M02930; Wuhan Boster Biological Technology, Ltd.) and their purity was determined by immunocytochemistry. The extracted cells were plated at $2 \times 10^{5} / \mathrm{ml}$ and rinsed in PBS three times for 2 min each time. Then, $4 \%$ paraformaldehyde was used to fix the cells at room temperature for $30 \mathrm{~min}$, followed by rinsing with PBS three 
times for 4 min each time, and placed in $0.3 \%$ Triton X-100 and soaked for $10 \mathrm{~min}$. Slides were washed again with PBS three times for 4 min each time, and put into $3 \% \mathrm{H}_{2} \mathrm{O}_{2}$ for $15 \mathrm{~min}$ to eliminate the influence of endogenous peroxidase. Then, 3\% goat serum was used for blocking at room temperature for $20 \mathrm{~min}$ to block the effect of non-specific binding sites. After blocking, the slides were rinsed with PBS three times for 2 min each time. Then, the slides were incubated with rabbit anti-rabbit NSE primary antibody (1:100; cat. no. ab180943; Abcam) and incubated overnight at $4^{\circ} \mathrm{C}$ on a shaker. The next day, cells were incubated with goat anti-rabbit IgG H\&L (HRP-conjugated) (1:500; cat. no. ab6721; Abcam) and incubated at room temperature for $1.5 \mathrm{~h}$. The sides were washed with PBS three times for 5 min each time, and then put into the enzyme-labeled avidin for $20 \mathrm{~min}$. The cells were incubated with DAB in the dark for $20 \mathrm{~min}$. After washing, the cells were stained with hematoxylin at room temperature for $10 \mathrm{sec}$. The slides were observed under an optical microscope, the field of view was selected at random and cell morphology was observed under the microscope, and the brown-stained cells were considered positive cells. The hippocampal neuron growth status was assessed via inverted microscopy (magnification, x200). The purity of the neurons was estimated using ImageJ v1.45s (National Institutes of Health).

Cellular activity assay. The hippocampal neurons were seeded into a 96-well plate at a density of 1,000/well, grown for 5 days and divided into the following groups: i) Control group (25 mmol/1 glucose); ii) $\mathrm{HG}$ group (45 mmol/1 glucose); iii) $\mathrm{HG}+$ low-dose blank serum group (45 mmol/1 glucose $+10 \%$ total volume of low-dose blank serum); iv) HG + medium-dose blank serum group (45 mmol/l glucose $+10 \%$ total volume of medium-dose blank serum); v) HG + high-dose blank serum group ( $45 \mathrm{mmol} / \mathrm{l}$ glucose $+10 \%$ total volume of high-dose blank serum); vi) HG + low-dose green tea serum group ( $45 \mathrm{mmol} / \mathrm{l}$ glucose $+10 \%$ total volume of low-dose green tea serum); vii) $\mathrm{HG}+$ middle-dose green tea serum group ( $45 \mathrm{mmol} / 1$ glucose $+10 \%$ total volume of medium-dose green tea serum); viii) $\mathrm{HG}+$ high-dose green tea serum group (45 mmol/l glucose $+10 \%$ total volume of high-dose green tea serum); ix) $\mathrm{HG}+\mathrm{SP}$ group [45 mmol/1 glucose + $10 \mu \mathrm{mol} / 1$ SP600125 (JNK inhibitor; Sigma-Aldrich; Merck KGaA)]; and x) $\mathrm{HG}+\mathrm{ML}-7$ group [45 mmol/1 glucose + $10 \mu \mathrm{mol} / \mathrm{l} \mathrm{ML-7}$ (MLCK inhibitor; Sigma-Aldrich; Merck KGaA)]. Cellular activity was assessed using Cell Counting Kit-8 (CCK-8) assays according to a previous study (25). Briefly, $10 \mu \mathrm{l} \mathrm{CCK}-8$ solution (cat. no. CK04; Dojindo Molecular Technologies, Inc.) was added to each well and the cultures were incubated in a water bath at $37^{\circ} \mathrm{C}$ for $2 \mathrm{~h}$. The optical density at $450 \mathrm{~nm}$ was then measured using a microplate reader and the activities of the cells in the different groups were calculated. All CCK-8 assays were performed in triplicate and repeated three times.

Cell apoptosis analysis. The cell culture medium used was the same as in Hippocampal neuronal cell culture and identification. Differentially treated hippocampal neuronal cells were cultured for $48 \mathrm{~h}$ and apoptosis was then assessed via incubation in the dark with an Annexin V-FITC apoptosis detection kit (Nanjing KeyGen Biotech Co., Ltd.) according to the manufacturer's protocol. The apoptosis rate was determined by calculating the percentage of early and late apoptotic cells. The cells were analyzed via fluorescence-activated cell sorting using a flow cytometer (Navios; Beckman Coulter, Inc.) and the results were analyzed using FlowJo v10 (FlowJo LLC) and GraphPad Prism 5 (GraphPad software, Inc.).

Diabetes induction. The present study established the type 1 diabetes rat model by the administration of an intraperitoneal injection of streptozotocin (STZ; Sigma-Aldrich; Merck KGaA). A total of 60 male SD rats were randomly divided into two groups: The negative control (NC) group and the model group. The rats in the NC group $(n=12)$ were fasted for $12 \mathrm{~h}$, intraperitoneally injected with citric acid-sodium citrate buffer $(0.1 \mathrm{~mol} / \mathrm{l}, \mathrm{pH} 4.2)$ and given daily administrations of an equal volume of sterile distilled water. The rats in the model group $(\mathrm{n}=48)$ were fasted for $12 \mathrm{~h}$ and administered an STZ solution (STZ dissolved in citric acid-sodium citrate buffer, $50 \mathrm{mg} / \mathrm{kg}$ ) via intraperitoneal injection to induce diabetes. As in our previous study (13), successful replication of this diabetes model was verified if the fasting blood glucose level at $72 \mathrm{~h}$ after STZ injection was $\geq 16.7 \mathrm{mmol} / \mathrm{l}$ and remained $>16.7 \mathrm{mmol} / \mathrm{l}$ for 3 consecutive weeks. The model group was then divided into four groups $(n=12)$ : i) The rats in the diabetes model (DM) group received an equal volume of distilled water; ii) the rats in the low-dose green tea extract group received $5 \mathrm{ml} / \mathrm{kg} /$ day green tea extract $+15 \mathrm{ml} / \mathrm{kg} /$ day sterile distilled water; iii) the rats in the medium-dose green tea extract group received $10 \mathrm{ml} / \mathrm{kg} /$ day green tea extract $+10 \mathrm{ml} / \mathrm{kg} /$ day sterile distilled water; and iv) the rats in the high-dose green tea extract group received $20 \mathrm{ml} / \mathrm{kg} /$ day green tea extract. Timed gavage was performed every day for 8 weeks to process the rats. The fasting blood glucose level and weight of the rats was measured every 3 weeks and the effect of green tea on the rat blood glucose levels was observed in the different groups.

Morris water maze test. In the 12th week after the diabetes model was established, the Morris water maze test was performed to assess the learning and memory abilities of the rats. According to a previous study (26), the basal swimming speed of the rats in each group was first tested. The DM group developed cognitive dysfunction at 12 weeks, as shown by the Morris water maze test, which included positioning navigation experiments and space exploration experiments. The water maze circular constant temperature pool had a diameter of $160 \mathrm{~cm}$ and a height of $50 \mathrm{~cm}$, while the circular platform had a diameter of $12 \mathrm{~cm}$ and its position was adjustable. In this experiment, the height was set to $30 \mathrm{~cm}$, and the water temperature was kept at $23 \pm 1^{\circ} \mathrm{C}$. The circular platform was placed in the center of the southeast quadrant of the pool $\sim 1 \mathrm{~cm}$ below the water surface. In the beginning, the rats were placed in the water from a position different from the edge of the northeast quadrant. The DigBehv animal behavior video tracking system (DigBehv-LM4; Shanghai Jiliang Software Technology Co., Ltd.) was used to track and record the time that the rats reached the platform, which was measured as the escape latency period. The rats that found the platform within $60 \mathrm{sec}$ were treated as a success. If the rat did not find the platform during this time, they were manually guided to the platform and kept there for $30 \mathrm{sec}$, and the escape latency of this rat was deemed to be $60 \mathrm{sec}$. The experiment was performed 
continuously for 5 days in total, four times a day. The escape latency was recorded on the last day of the experiment and the mean was calculated. During the space exploration experiment on the 6th day of the experiment, most conditions were left unchanged, but the platform in the pool was removed and the rats were allowed to enter the pool at any one of the marked points. The program recorded the rats' swimming trajectory within $60 \mathrm{sec}$ and recorded their swimming tracks in $60 \mathrm{sec}$. The number of crossings over the original platform during $60 \mathrm{sec}$ was considered to evaluate the space exploration ability of the rat.

Hematoxylin and eosin $(H \& E)$ staining. Morphological changes in the hippocampus of the rats in the different groups were observed via H\&E staining. The hippocampal tissue was dissected and paraffin-embedded sections were prepared (slice thickness, $4 \mu \mathrm{m}$ ). The slices were dehydrated by ethanol gradient (80,95 and $100 \%$ ethanol), soaked in xylene two times for $5 \mathrm{~min}$ each time to make the slices transparent, stained with hematoxylin for $1 \mathrm{~min}$ and stained with eosin solution for $1 \mathrm{~min}$ at $25^{\circ} \mathrm{C}$, and finally mounted with neutral gum. Slices were observed with a Nikon Eclipse E100 microscope (Nikon Corporation).

Transmission electron microscopy (TEM) of the hippocampal tissue ultrastructure. Rats were anaesthetized via intraperitoneal injection of sodium pentobarbital $(100 \mathrm{mg} / \mathrm{kg})$ and subjected to intracardiac perfusion-fixation using a solution of $0.9 \%$ sodium chloride (VWR International, LLC), then all rats were sacrificed via cervical dislocation. and $4 \%$ paraformaldehyde (Sigma-Aldrich; Merck KGaA) in 0.1 M PBS (pH 7.4). The hippocampal tissue was obtained and placed in fixative. Shanghai Fucheng Biotechnology Co., Ltd., processed the samples and the samples were processed according to the following steps. The samples (slice thickness, $70 \mathrm{~nm}$ ) were fixed at room temperature in $2.5 \%$ glutaraldehyde ( $\mathrm{pH} 7.4)$ for $2 \mathrm{~h}$ and embedded in agarose with low melting point. After washing three times with $0.1 \mathrm{M}$ PBS ( $\mathrm{pH} 7.2)$, samples were fixed in $1 \%$ osmic acid at $4^{\circ} \mathrm{C}$ for $2 \mathrm{~h}$. Then, the samples were dehydrated in a graded series of ethanol. Subsequently, the samples were embedded in Epon-Araldite resin for penetration and placed in a model for polymerization. Ultrathin sections were collected for microstructure analysis. Following counterstaining with $3 \%$ uranyl acetate and $2.7 \%$ lead citrate, slices were observed with a HT7800 transmission electron microscope (Hitachi, Ltd.).

Analysis of apoptosis in hippocampal tissue. The degree of apoptosis in the hippocampal tissues from each group was detected through terminal deoxynucleotidyl transferase-mediated dUTP nick end-labelling (TUNEL) assays using a TUNEL Apoptosis kit (Nanjing KeyGen Biotech Co., Ltd.) according to the manufacturer's recommended protocol. The positive cells in each group were counted under a microscope (a brown color indicated positive apoptotic cells) and the degree of apoptosis in the hippocampus was assessed using ImageJ software v1.6.0 (National Institutes of Health).

Western blot analysis. Following collection of hippocampal tissue and hippocampal neurons, protein was extracted using a protein extraction kit (Nanjing KeyGen Biotech Co., Ltd.). Protein concentration was determined using a BCA assay (Pierce; Thermo Fisher Scientific, Inc.). The protein samples $(20 \mu \mathrm{g})$ were separated via SDS-polyacrylamide on $10 \%$ gels. The target protein was then transferred to a $0.45 \mu \mathrm{m}$ Immobilon-P PVDF membrane (cat. no. IPVH00010; Merck $\mathrm{KGaA}$ ) via the wet transfer method and the membrane was blocked with $5 \%$ skimmed milk for $2 \mathrm{~h}$ at room temperature and incubated overnight at $4^{\circ} \mathrm{C}$ on a shaker with the following antibodies: Rabbit anti- $\beta$-actin $(1: 5,000$; cat. no. ab8227; Abcam), rabbit anti-JNK (1:2,000; cat. no. 9252S; Cell Signaling Technology, Inc.), rabbit anti-p-JNK (1:2,000; cat. no. 4668; Cell Signaling Technology, Inc.), rabbit anti-MLCK (1:5,000; cat. no. ab76092; Abcam), rabbit anti-Bcl-2 (1:1,000; cat. no. ab182858; Abcam) and rabbit anti-Bax (1:1,000; cat. no. ab32503; Abcam). The membrane was then subjected to three 10 - $\mathrm{min}$ rinses in TBS with $0.1 \%$ Tween-20 buffer (TBST), incubated with HRP-conjugated sheep anti-rabbit IgG (1:20,000; cat. no. 7074; Cell Signaling Technology, Inc.) secondary antibody for $2 \mathrm{~h}$ at room temperature and rinsed three times for $10 \mathrm{~min}$ in TBST buffer. The ECL method (Amersham; Cytiva) was used to display the signals from the target proteins and a gel imaging system was used for image collection. The image grey values were analyzed and compared using ImageJ software v1.6.0 (National Institutes of Health) to calculate the relative expression levels. Western blot analyses were repeated at least three times for each protein.

Statistical analysis. All data are expressed as the mean \pm standard deviation. SPSS v19.0 software (IBM Corp.) was used for data analysis. Inter-group variation was measured using a one-way ANOVA, followed by a Tukey's post hoc test. $\mathrm{P}<0.05$ was considered to indicate a statistically significant difference.

\section{Results}

HG leads to increased apoptosis in hippocampal neurons. The present study first prepared primary cultured hippocampal neurons and detected the purity of the cells by staining for NSE (Fig. 1A). Hippocampal neuronal apoptosis was detected in the HG group (Fig. 1B). The HG group demonstrated a significantly increased apoptosis rate compared with the control group. Cellular activity was also detected using a CCK- 8 assay and the results demonstrated that the cellular activity in the HG group was significantly decreased (Fig. 1C). In addition, the expression of the apoptosis-related proteins Bcl-2 and Bax in the two groups was determined and the HG group demonstrated decreased Bcl-2 levels and increased Bax levels compared with the control group (Fig. 1D).

Increased apoptosis of hippocampal neurons in diabetic rats with cognitive dysfunction. The present study successfully replicated diabetes, as shown by the fact that blood glucose levels $\geq 16.7 \mathrm{mmol} / \mathrm{l}$ were maintained for $>3$ weeks (Fig. 2A) and the diabetes model was maintained for 2 months. Prior to cognitive function tests, the basal swimming speed of the rats in each group was tested and no differences were found between groups (Table SI). The Morris water maze test demonstrated that the rats in the diabetic group exhibited significant declines 
A

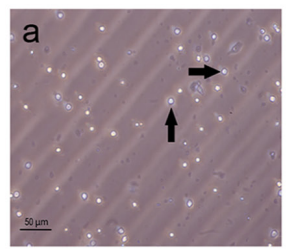

B
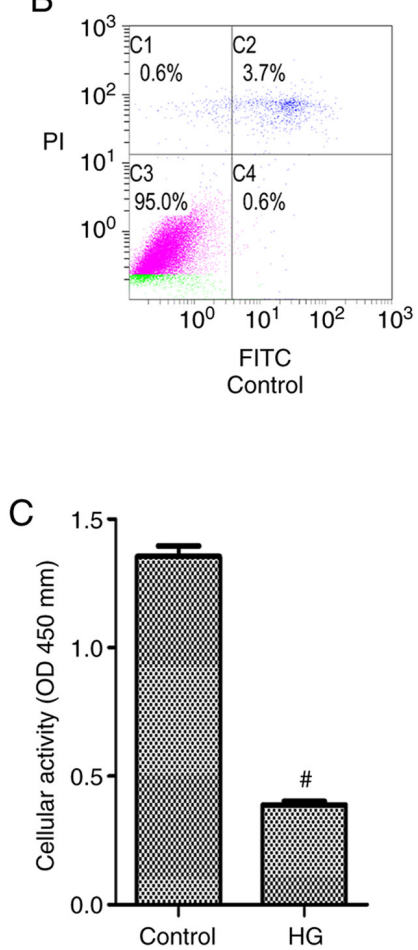
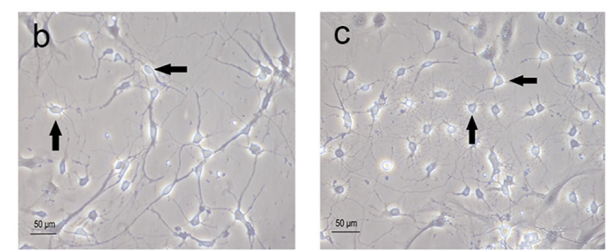
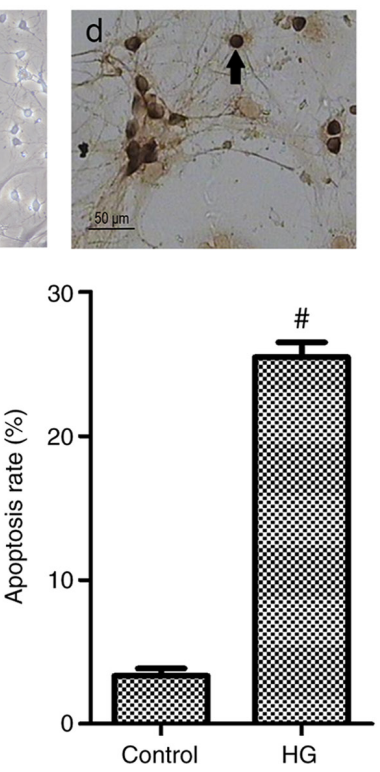

D
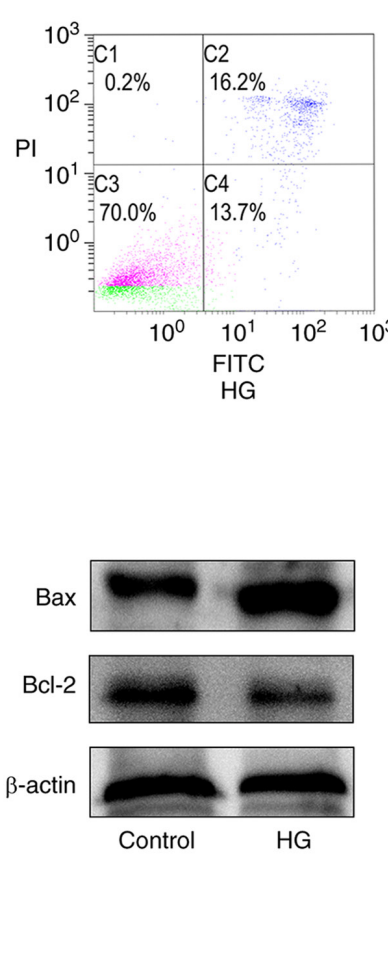

$\mathrm{HG}$

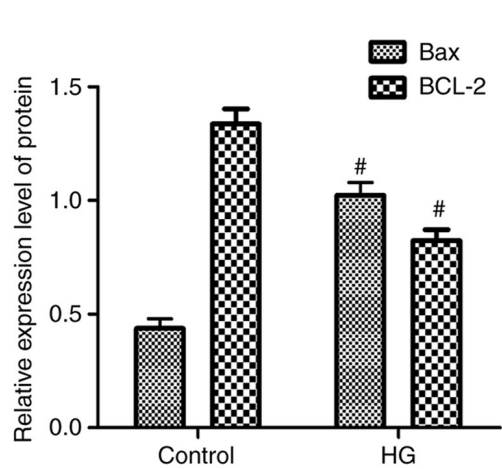

Figure 1. HG leads to increased apoptosis in hippocampal neurons. (A-a) Hippocampal neuron culture day 1 (magnification, x200). (A-b) Hippocampal neuron culture day 3 (magnification, x200). (A-c) Hippocampal neuron culture day 5 (magnification, x200). (A-d) Hippocampal neuronal cells were identified following immunocytochemical staining with anti-neuron-specific enolase (magnification, x400) black arrow indicates hippocampal neurons. (B) Apoptosis of hippocampal neurons in the control and HG groups. (C) Hippocampal neuron activity was evaluated using Cell Counting Kit-8 assay data. (D) Expression of the apoptosis-associated proteins Bcl-2 and Bax in hippocampal neurons in the control and HG groups. Each data point represents the mean \pm standard deviation ( $\mathrm{n}=3$ ). ${ }^{\#} \mathrm{P}<0.05$ vs. the control group. OD, optical density; HG, high-glucose.

in both learning and memory (Fig. 2B and C; Table SII), as illustrated by the lines in Fig. S1C, which showed the movement of the rats and the decreased cognitive function of the diabetic rats. Rat hippocampal tissues were then collected for observation and H\&E staining (Fig. 2D). Specifically, the morphology of the neurons in the CA1 subfield of the hippocampi of rats was detected by H\&E staining. Analysis of the NC group demonstrated that the nerve cells in each structure were complete, but staining of the hippocampal tissues of the DM group revealed that the neurons adopted a disordered arrangement and that the structure of the vertebral body and nucleus of nerve cells was destroyed. Furthermore, the number of hippocampal neurons was reduced and vacuoles were observed in the cytoplasm. The hippocampal neuron ultrastructure in the NC group was also observed via TEM (Fig. 2E) and it was noted that the morphological structure of the hippocampus was intact, the nuclei were large, the cytoplasm was rich in content and intact, the mitochondria were numerous and well-arranged and the endoplasmic reticulum was regular. By contrast, analysis of the diabetic rats revealed that the neurons were damaged, the mitochondria number was reduced and the structure was destroyed. TUNEL assays were used to detect apoptosis of hippocampal neurons and the CA1 region of the hippocampus selected for observation and evaluation of neuronal apoptosis; a brown-black color indicated apoptotic cells. Assessment of the NC group revealed intact cells in the hippocampal tissue, few apoptotic cells and a regular arrangement of normal cells; however, more brown-black cells, which indicated apoptosis, were observed in the DM group (Figs. 2F and S2A; Table SIII). To further verify these changes, western blot analysis was performed and the results confirmed increased expression of the apoptosis-related protein Bax and reduced expression of the apoptosis-related protein Bcl-2 in the DM group (Fig. 2G). Taken together, these results showed that increased apoptosis of hippocampal neurons led to decreased cognitive function in diabetic rats.

Identification of six components in green tea serum. To further study the components in green tea serum, blank serum (Fig. 3A), mixed standards (Fig. 3B) and green tea serum (Fig. 3C) were assessed via HPLC. A total of six components were detected in green tea serum: Gallic acid, catechin, caffeine, EGCG, EC and ECG at mass concentrations of 3.6, $4.2,54.6,22.8,3.8$ and $4.8 \mu \mathrm{g} / \mathrm{ml}$, respectively. 
A

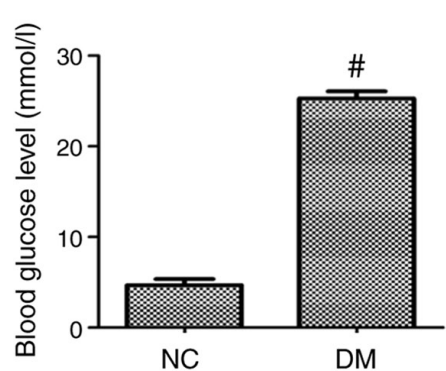

B

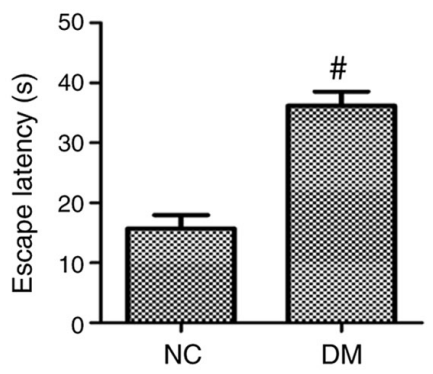

C

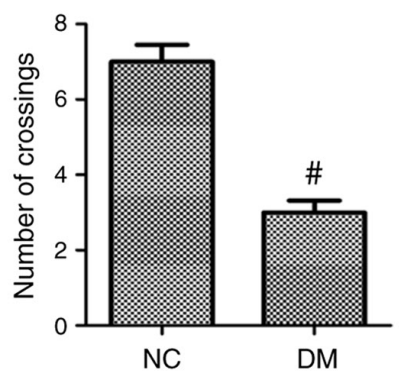

D

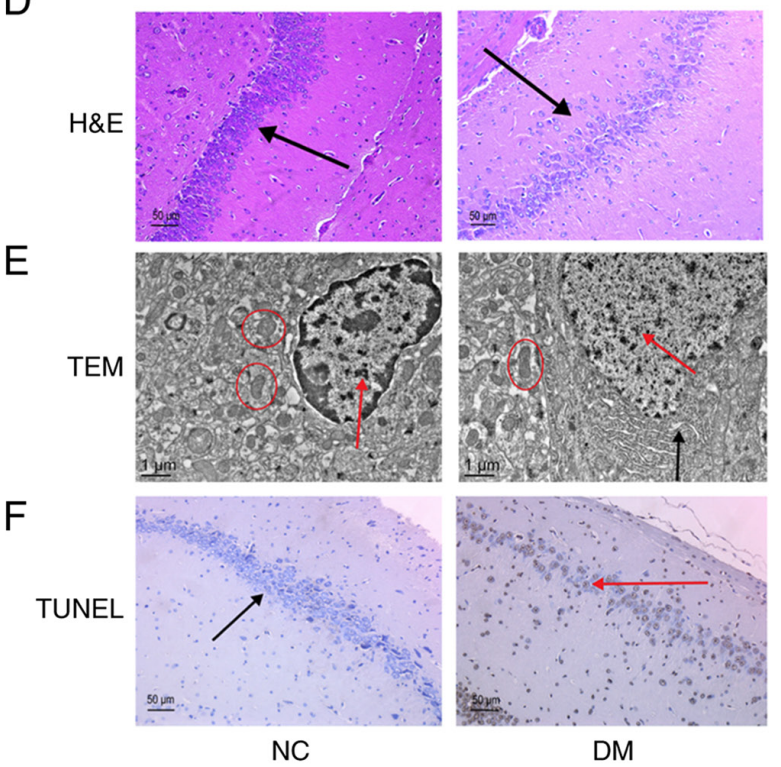

G
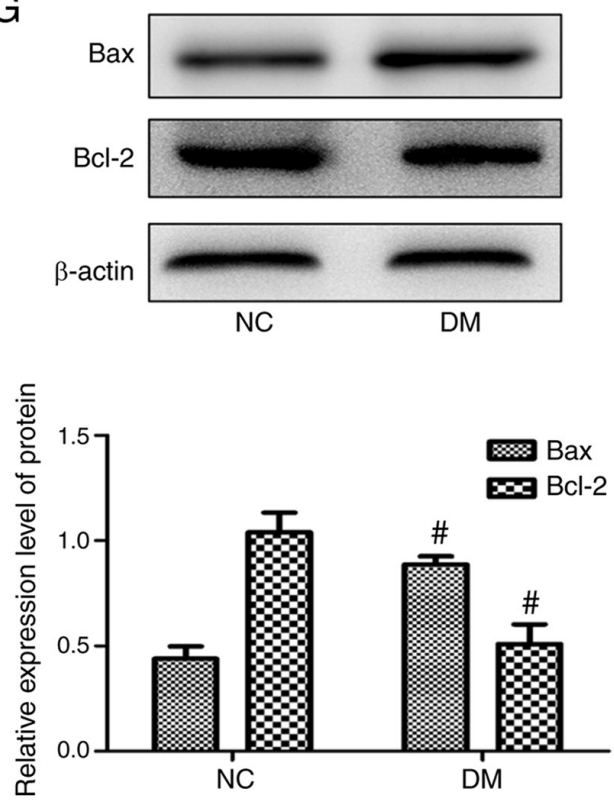

Figure 2. Increased apoptosis of hippocampal neurons in rat models of diabetic cognitive dysfunction. (A) Blood glucose levels in different groups in the 12th week. (B) Escape latency in the Morris water maze test in the 12th week. (C) Number of rat crossings in the Morris water maze test in the 12th week. (D) H\&E staining showing the morphology of neurons in the CA1 subfield of the hippocampus (magnification, x200), black arrow indicates hippocampal neurons. (E) TEM observation of hippocampus ultrastructure (magnification, x10,000), red arrows indicate nuclei; black arrows indicate endoplasmic reticulum; red circles indicate mitochondria. (F) TUNEL was used to detect apoptosis in the hippocampus (magnification, x200), red arrow indicates apoptotic hippocampal neurons; black arrow indicates normal hippocampal neurons. (G) Expression of apoptosis-associated proteins Bcl-2 and Bax in hippocampal neurons examined by western blot analysis. Each data point represents the mean \pm standard deviation $(n=3)$. ${ }^{~} \mathrm{P}<0.05$ vs. the NC group. H\&E, hematoxylin and eosin; TEM, transmission electron microscopy; NC, negative control; DM, diabetes model.

Green tea serum inhibits Bax to protect hippocampal neurons from $H G$-induced apoptosis. First, data were obtained from an in vitro cell culture study in which no difference was found in apoptotic rate among the low-, medium- and high-dose blank serum groups and the HG group. In the low-, medium- and high-dose green tea serum groups, the apoptotic rate demonstrated a downward trend and the highest dose of green tea serum exerted the most significant effect (Fig. 4A). In addition, no difference in cellular activity was found among the low-, medium- and high-dose blank serum groups and the HG group and the cellular activity was increased in the low-, mediumand high-dose green tea serum groups (Fig. 4B). It was also found that the expression of the apoptotic proteins $\mathrm{Bcl}-2$ and Bax was not significantly different between the low-, mediumand high-dose blank serum groups and the HG group, but the high-dose green tea serum group exhibited decreased Bax levels and increased Bcl-2 levels compared with the HG group (Fig. 4C). Overall, these results confirmed that green tea serum could inhibit Bax to protect hippocampal neurons from HG-induced apoptosis.
Green tea improves cognitive dysfunction in diabetic rats by inhibiting hippocampal neuronal apoptosis. To further probe the effects of green tea in vivo, green tea extract was administered to diabetic rats and found that green tea reduced blood glucose levels (Figs. 5A and S1A) and slowed the weight loss in these rats (Fig. S1B). The results of the Morris water maze test demonstrated that green tea improved the learning and memory abilities of the diabetic rats (Fig. 5B and C), as illustrated by the lines in Fig. S1C, which show the movement of the rats. H\&E staining of hippocampal tissues from the green tea-treated rats demonstrated that the hippocampal neuron cell structure was more complete, the nucleolus was clearer and the number of cells was higher than observed in the other groups (Fig. 5D). Similar results were obtained by TEM, which demonstrated that green tea protected against changes to the ultrastructure of hippocampal neurons (Fig. 5E). The CA1 region of the hippocampus was subsequently selected for observation and evaluation of neuronal apoptosis in the various groups. The low-, medium- and high-dose green tea groups exhibited decreased hippocampal neuronal apoptosis 

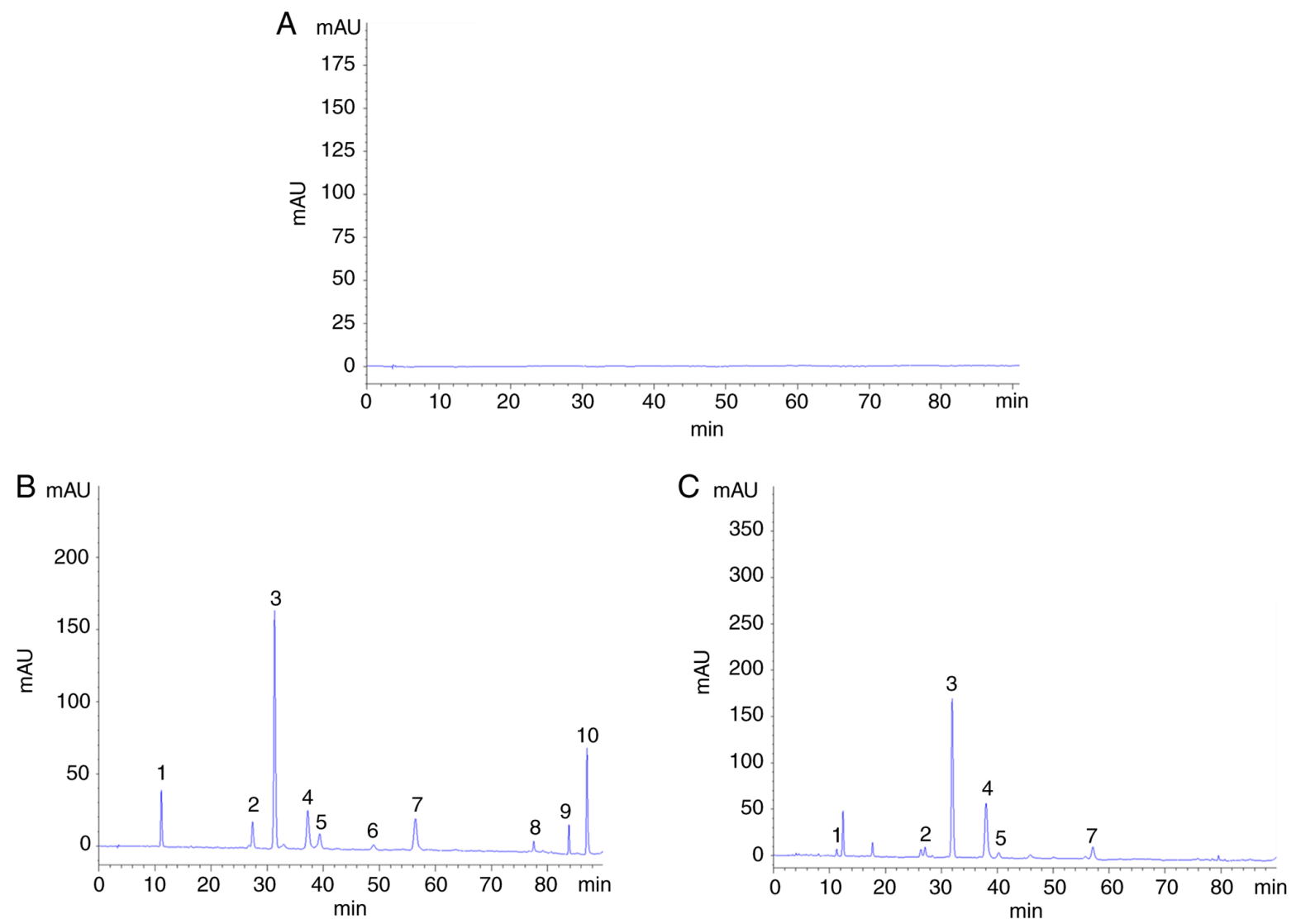

Figure 3. Green tea components in green tea serum. (A) The 10 tea components in blank serum. (B) The 10 green tea ingredients in the mixed standard. (C) The 10 green tea ingredients in tea serum. 1 , gallic acid; 2 , catechin; 3 , caffeine; 4 , epigallocatechin catechin gallate; 5 , epicatechin; 6, gallic acid catechin gallate; 7, epicatechin gallate; 8 , rutin; 9, quercetin; 10, kaempferol.

and the high-dose green tea group had the lowest apoptosis rate and presented regularly arranged cells (Figs. 5F and S2). Taken together, these results indicated that green tea can reduce blood sugar levels and improve cognitive function in diabetic rats.

Green tea protects against hippocampal neuronal apoptosis via the JNK/MLCK pathway. The JNK signaling pathway serves an important role in apoptosis and our previous study found that MLCK serves a role in hippocampal neuronal apoptosis in DE (14). Therefore, the present study further examined the relationship between the JNK pathway and MLCK. First, it was found that the JNK signaling pathway regulates MLCK expression and that activation of the JNK pathway with HG promoted MLCK expression in hippocampal neurons (Fig. 6A). The mechanism through which green tea protects hippocampal neurons from apoptosis was further clarified by flow cytometry and the results demonstrated that the $\mathrm{HG}+\mathrm{SP}$ and $\mathrm{HG}+$ green tea serum groups exhibited significantly reduced apoptotic rates (Fig. 6B). CCK-8 assays demonstrated that cellular activity was increased in the $\mathrm{HG}$ $+\mathrm{SP}$ and $\mathrm{HG}+$ green tea serum groups (Fig. 6C). Western blot analysis demonstrated that the green tea serum groups exhibited decreased p-JNK, MLCK and Bax expression and increased Bcl-2 expression (Fig. 6D) and similar results were detected in model rats treated with green tea. The most significant decreases in p-JNK, MLCK and Bax expression and the most significant increases in $\mathrm{Bcl}-2$ expression among the green tea-treated groups were found in the high-dose green tea group (Fig. 6E). Taken together, these results suggested that green tea protects against hippocampal neuronal apoptosis by inhibiting activation of the JNK/MLCK pathway in DE.

\section{Discussion}

The pathogenesis of DE is complicated and it is currently difficult to identify effective therapeutic targets for DE (27). It is becoming epidemiologically clear that the intake of green tea suppresses cognitive decline. A previous study indicated that green tea intake might reduce the risk of dementia, Alzheimer's disease, mild cognitive impairment and severe cognitive impairment (28), but the exact mechanism of action of green tea remains unclear. Previous studies have found that the occurrence of diabetic cognitive dysfunction is associated with apoptosis, oxidative stress, perturbed calcium homeostasis, altered energy metabolism and microvascular disease (29-31). Our previous study found that hippocampal neuronal apoptosis is an important mechanism leading to DE development (14). Hyperglycemia can lead to the occurrence of downstream apoptosis through activation of the JNK signaling pathway and activation of this pathway can induce the mitochondrial apoptotic pathway, which is involved in the pathogenesis of diabetic cognitive dysfunction. As mentioned in a literature review, the JNK signaling pathway serves not only an important role in apoptosis, but also a substantial role in the pathogenesis of neurological disorders (32). JNK, an 
A

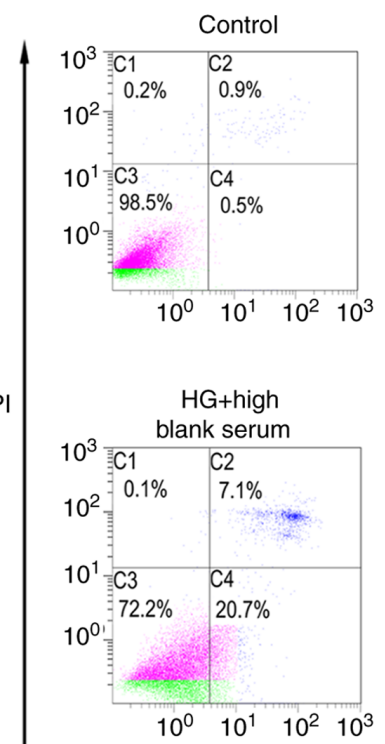

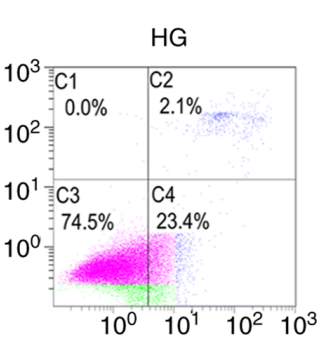
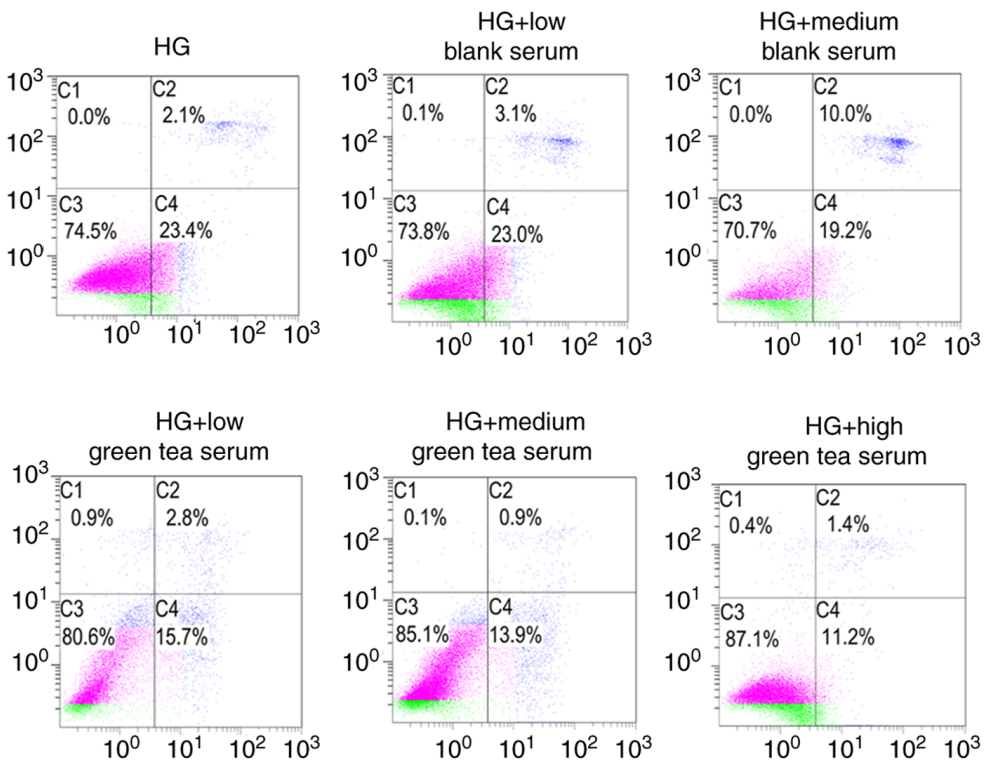

FITC
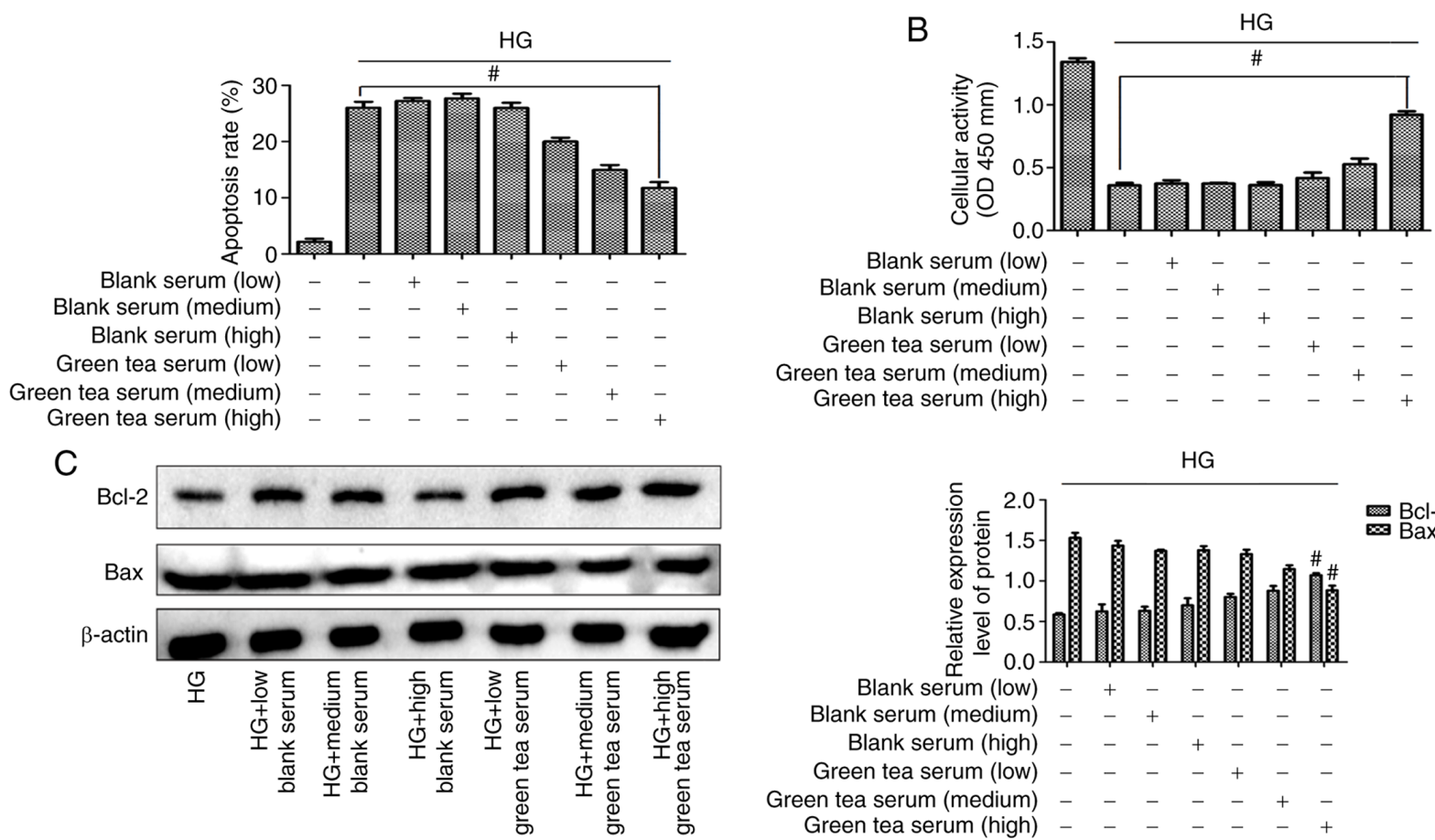

Green tea serum (high)

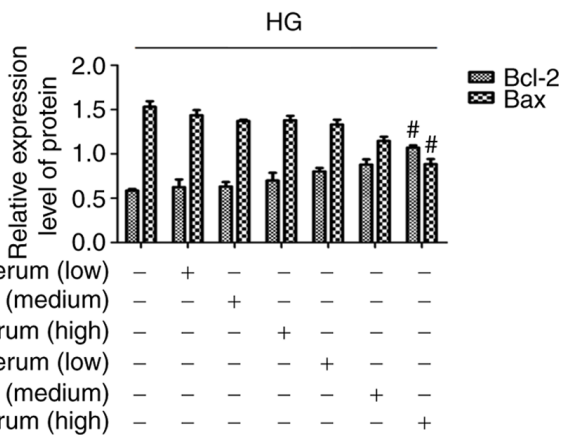

Figure 4. Green tea serum inhibits Bax to protect against HG-induced hippocampal neuronal apoptosis. (A) Flow cytometry assay was performed to detect hippocampal neuronal apoptosis. (B) The activity was tested in each group using Cell Counting Kit-8 assays. (C) Expression of apoptosis-related proteins were detected via western blotting. Each data point represents the mean \pm standard deviation. ${ }^{\#} \mathrm{P}<0.05$ vs. HG group. HG, high-glucose.

important member of the MAPK family, has three isoforms, JNK1, JNK2 and JNK3 (16). The JNK pathway can activate a variety of signaling factors, including TNF- $\alpha$, epidermal growth factor, $\mathrm{G}$ protein-coupled receptors and stress, which results in cytochrome c release and subsequent activation of the mitochondrial apoptosis pathway and the occurrence of apoptosis (33). Several studies have shown that the JNK signal transduction pathway is involved in the neurotoxic damage induced by amyloid $\beta$ and closely associated with the apoptosis of hippocampal neurons $(34,35)$. Dendropanax morbifera has been reported to improve diabetic cognitive dysfunction by reducing the level of oxidative stress and inhibiting the JNK signaling pathway (36). A recent study found that Nuanxin Capsules, which are a Traditional Chinese Medicine that have a protective effect against heart failure, can reduce Bcl-2 expression and increase Bax expression by inhibiting activation of the JNK pathway, ultimately inhibiting apoptosis induced via the mitochondrial pathway (37). The present study demonstrated that hyperglycemia promoted hippocampal neuron apoptosis by activating the JNK signaling pathway, which serves a central role in DE onset and development. The JNK signaling pathway regulates neuronal apoptosis during the development of diabetic encephalopathy, but a previous study found that activation of the PI3K/Akt/GSK3 $\beta$ pathway 
A
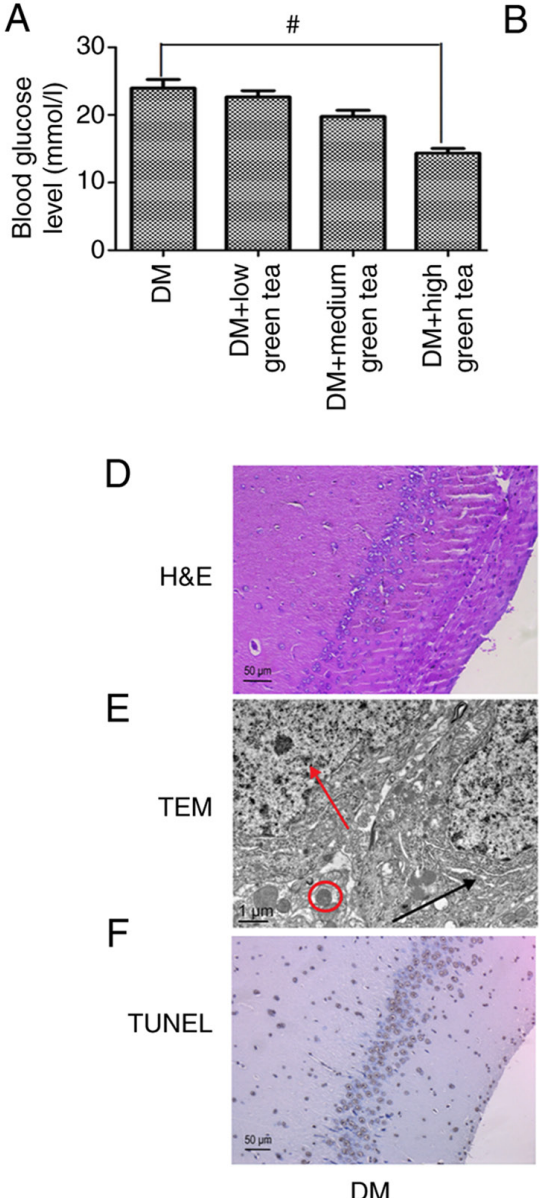

B

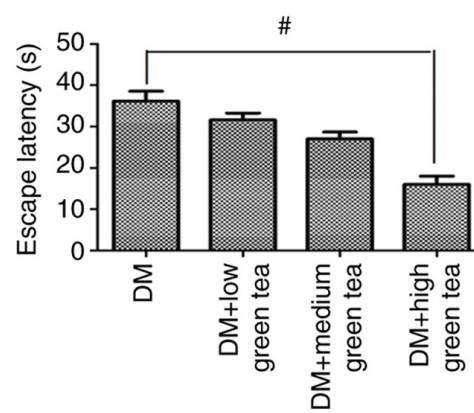

C

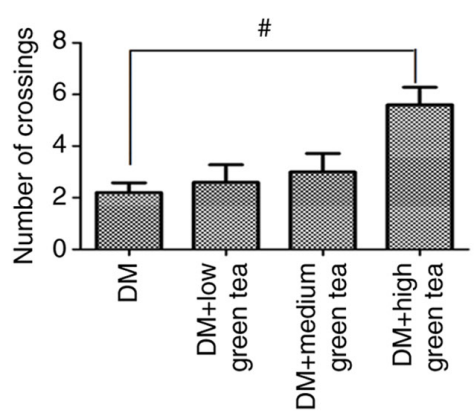

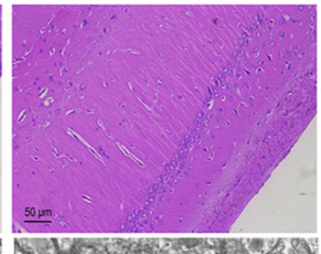
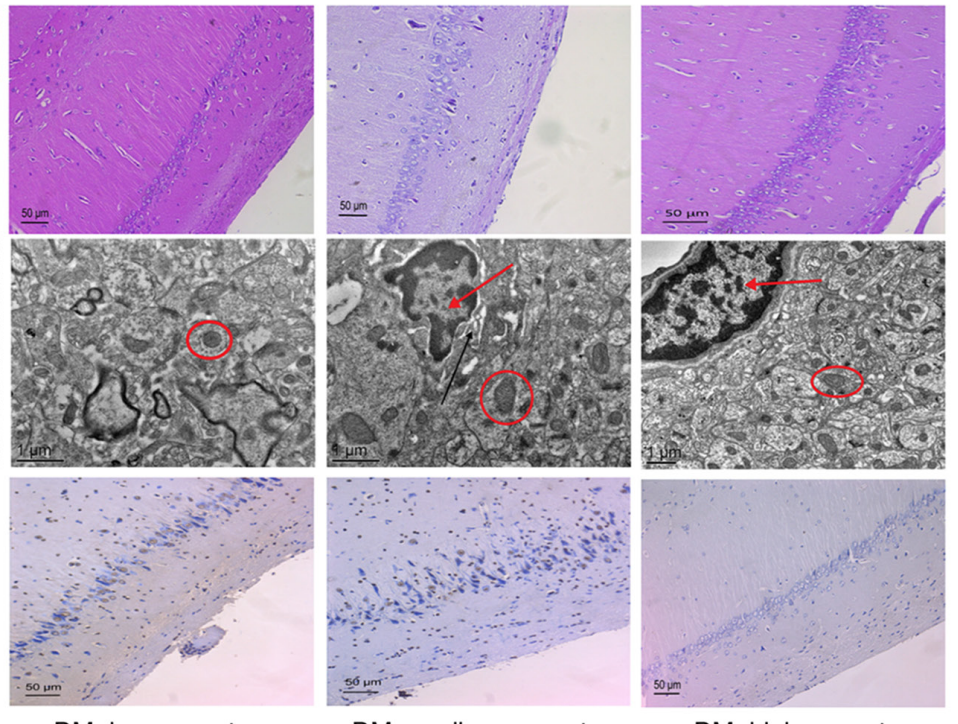

$\mathrm{DM}+$ low green tea
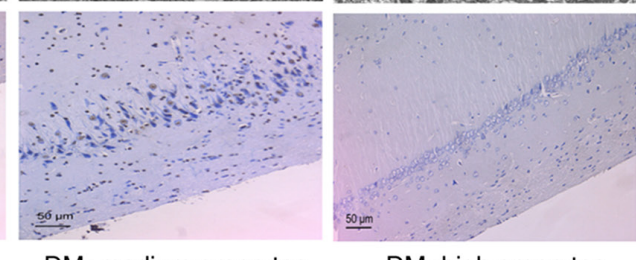

$\mathrm{DM}+$ medium green tea

Figure 5. Green tea improves cognitive dysfunction in diabetic rats by inhibiting hippocampal neuronal apoptosis. (A) Blood glucose level of rats was tested in the 12th week. (B) Escape latency in the Morris water maze test in each group in the 12th week. (C) Number of rat crossings in the Morris water maze test in the 12th week. Each data point represents the mean \pm standard deviation. ${ }^{~} \mathrm{P}<0.05$ vs. the DM group. (D) Histological observation of hippocampal tissues following H\&E staining (magnification, x200). (E) Effects of green tea on the ultrastructural features of hippocampal neurons visualized with TEM (magnification, x10,000), red arrow indicates nuclei; black arrow indicates endoplasmic reticulum; red circle indicates mitochondria. (F) TUNEL was used to detect apoptosis in the hippocampus (CA1; magnification, $\mathrm{x} 200)$. DM, diabetes model; H\&E, hematoxylin and eosin; TEM, transmission electron microscopy.

can improve tau hyperphosphorylation and axon damage and thereby exert cognitive protection (38). In short, cognitive dysfunction caused by DE is the result of multiple pathways. The present study only studied apoptosis of hippocampal neurons and further evidence is needed.

MLCK is an important functional protein that regulates smooth muscle contraction. Activated MLCK phosphorylates the myosin light chain and phosphorylated myosin light chain induces smooth muscle contraction (8). MLCK was the first CaM-dependent kinase to be discovered and free $\mathrm{Ca}^{2+}$ obtained after the binding of MLCK to calmodulin activates MLCK, which in turn activates contractile proteins (39). MLCK expression is increased in the hippocampi of rats with diabetic cognitive dysfunction. Inhibition of MLCK expression has also been found to decrease the hippocampal neuronal apoptosis rate, but the underlying mechanism remains unknown (10). At present, few studies have investigated the MLCK and JNK pathways, but a previous study found that loss of MLCK leads to disruption of cell-cell adhesion and invasive behavior in breast epithelial cells via increased expression of JNK signaling (40). Thus, the present study further investigated the relationship between MLCK and the JNK pathway. It used specific protein inhibitors (SP600125 and ML-7) that act on hippocampal neurons and found that activation of the JNK pathway promoted MLCK expression, leading to changes in the expression of the apoptosis-related proteins Bax and Bcl-2 and ultimately affecting the occurrence of apoptosis. Although the results indicated that MLCK is regulated by the JNK pathway, which is inconsistent with previous studies, the present study might have some limitations. For example, it did not directly elucidate the relationship between JNK and MLCK at the gene level. Despite its best attempts, no method to address the low transfection efficiency of hippocampal neurons was found, which is an important issue to address in future studies and it is intended to further verify the regulatory relationship between JNK and MLCK through immunoprecipitation experiments. HT-22 cells were selected as a model cell line to optimize the in vitro experiments to solve the problem of low transfection efficiency. The authors are currently establishing hippocampus-specific MLCK-knockout mice to explore the precise mechanisms underlying these effects.

Current pharmacological therapies for diabetes have failed to prevent DE development and there is an urgent need for alternative treatment strategies. Green tea is one of the most frequently and heavily consumed beverages worldwide and 
A

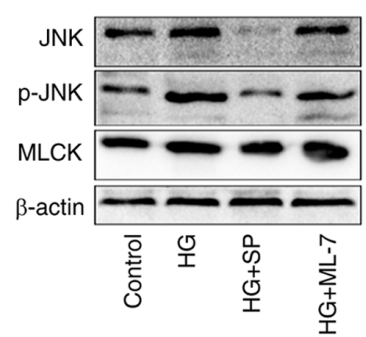

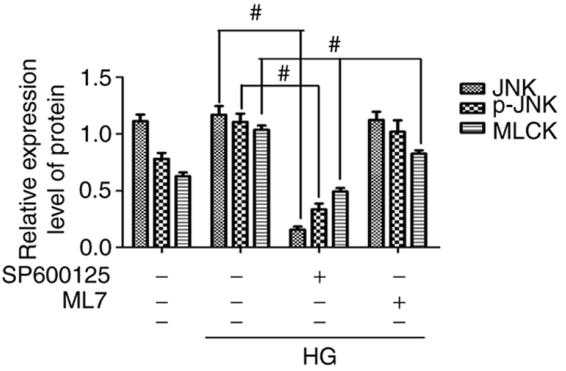

B
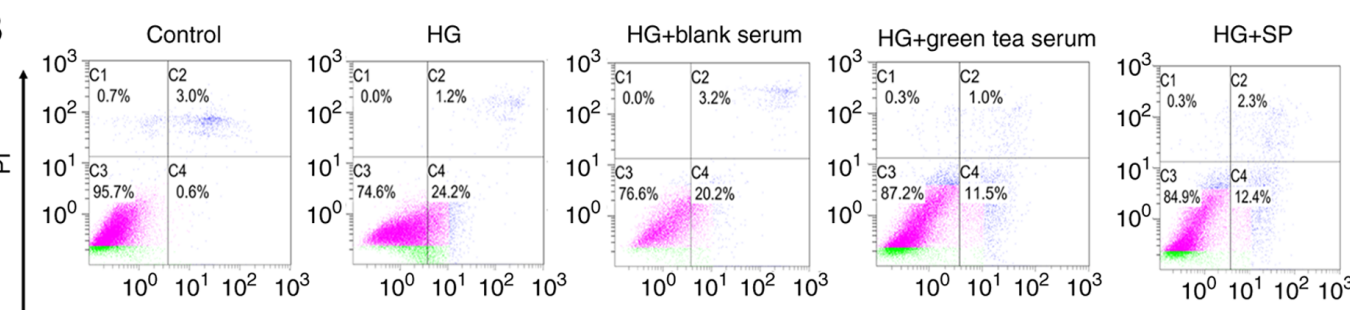

FIFC

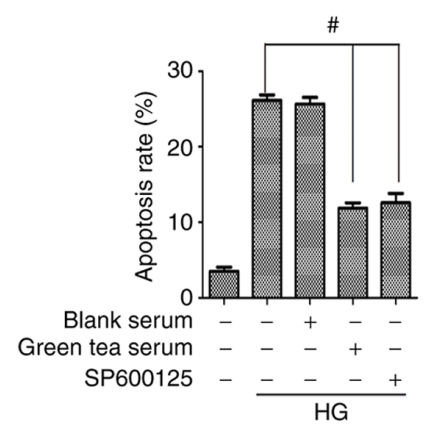

C

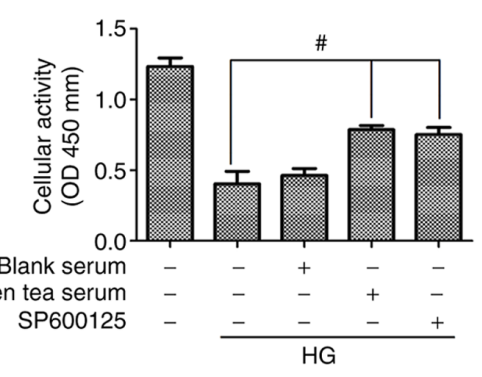

D

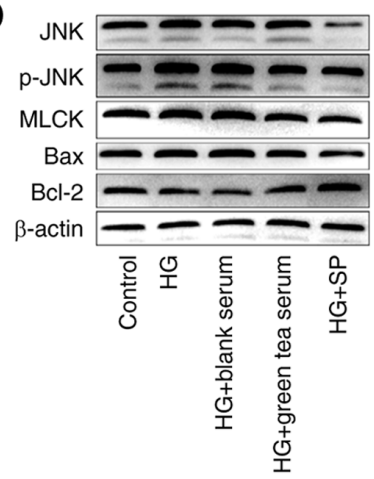

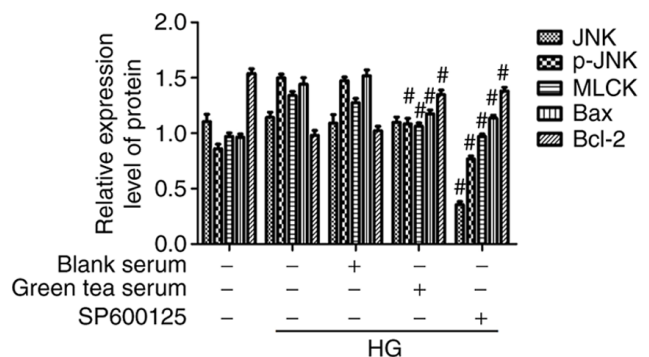

E

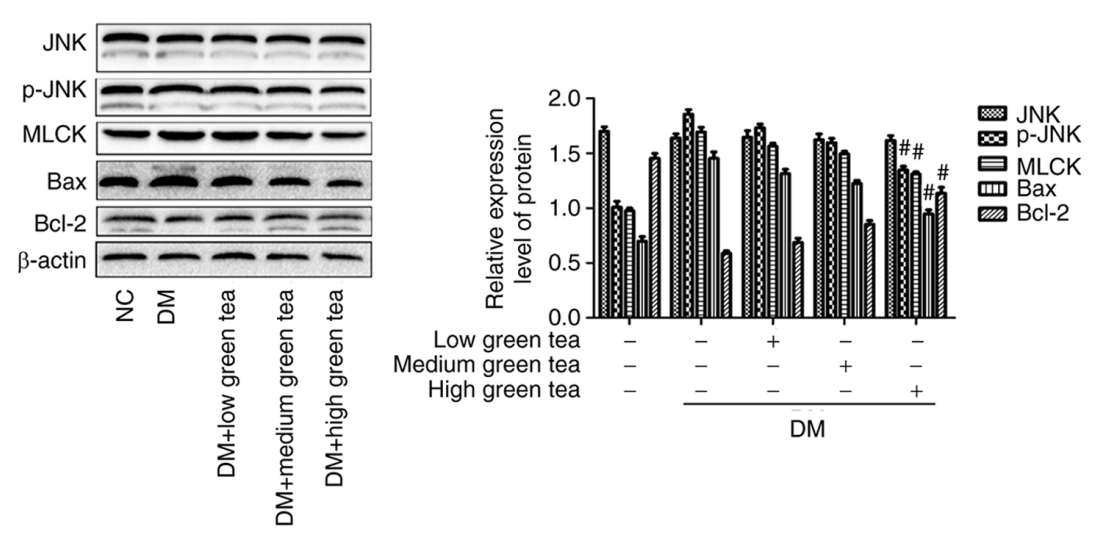

Figure 6. Green tea protects against hippocampal neuronal apoptosis via the JNK/MLCK pathway. (A) Relationship between the JNK pathway and MLCK was explored via western blotting. (B) Flow cytometry was used to detect apoptosis in each group. (C) Cell activity in each group was tested using Cell Counting Kit- 8 assays. (D) JNK, p-JNK, MLCK, Bcl-2 and Bax expression was detected in vitro. Each data point represents the mean \pm standard deviation. ${ }^{\#} \mathrm{P}<0.05$ vs. the HG group. (E) JNK, p-JNK, MLCK, Bcl-2 and Bax expression detected in vivo via western blotting. Each data point represents the mean \pm standard deviation. "P<0.05 vs. the DM group. MLCK, myosin light chain kinase; HG, high-glucose; p-, phosphorylated; DM, diabetes model; SP, SP600125; OD, optical density; NC, negative control. 
retains more of the natural ingredients in tea than other types of tea (20) Compared with other teas, green tea contains more tea polyphenols, alkaloids, theanine and other biologically active substances, the most abundant of which are EGCG and caffeine (41). For the present study, a seropharmacological method was selected to study the effects of green tea on neurons. Compared with other methods, seropharmacology, a novel method for pharmacological study of Traditional Chinese Medicine (42), has improved effects on assessing the actual effects of a drug on the body and the pharmacological effects of the drug itself and its metabolites (43). Therefore, green tea serum was obtained from green tea-gavaged rats via seropharmacological methods according to the literature and six active components in green tea serum were detected using HPLC. Among these components, EGCG and caffeine were present at the highest concentrations, which is similar to the levels of the components found in green tea.

Dietary factors might serve a role in prevention of cognitive dysfunction and among these factors, beverages are considered useful because their intake does not substantially affect other dietary habits and is more acceptable (1). Green tea is one of the most common beverages consumed worldwide and serves a role in neurodegenerative diseases and improving cognitive function (21). Tea polyphenols, alkaloids and theanine, which are the main ingredients of green tea, penetrate the blood-brain barrier and enter the brain tissue (44). A previous study found that green tea catechins have antioxidant activity, which can improve cognitive function in patients with Alzheimer's disease by scavenging free radicals (45). Green tea polyphenols reduce the inflammation associated with encephalopathy and improve cognitive ability by inhibiting NFK- $\beta$ activation (46). Treatment with EGCG in vitro $(10 \mathrm{nmol} / \mathrm{l})$ and in vivo $(2.5 \mathrm{mg} / \mathrm{kg}$; equivalent human dose of $0.27 \mathrm{mg} / \mathrm{kg}$ ) significantly improves the survival rate of hippocampal neurons and the cognitive function of the organism, potentially by influencing the activity of nerve cells by activating the PI3K/AKT signaling pathway (47). The present study found that intervention with green tea decreased blood glucose levels in diabetic rats and improved their memory and learning abilities. In addition, the cell experiments demonstrated that green tea serum protected against HG-induced apoptosis of hippocampal neurons by reducing the expression of the pro-apoptotic gene Bax and increasing cell viability. Therefore, HG-induced hippocampal neurons were treated with a JNK inhibitor and green tea serum to explore the mechanism through which green tea protects neurons. The experimental results of the present study demonstrated that green tea serum significantly inhibited p-JNK expression and JNK signaling pathway activation, which resulted in protection of cells from apoptosis. Similar results were obtained using animal models. In summary, the findings of the present study suggested that the beneficial effects through which green tea consumption improves cognitive function in diabetic rats might be associated with decreased hippocampal neuronal apoptosis. Afzal et al (48) reported that EGCG, the main component of green tea catechins, exerts neuroprotective effects because EGCG inhibits amyloid- $\beta$ aggregation. The present study provided the first demonstration of the effect of green tea in improving cognitive function in diabetes and clarifies the mechanism of action through which green tea protects cognitive function through anti-apoptotic activity. However, the present study has a number of limitations. First, it is not known whether the improvement in cognitive function occurred due to a combination of multiple ingredients in green tea or a single ingredient and thus it is planned to study the individual components of green tea to observe their effects on DE and provide further evidence to elucidate the mechanism through which green tea protects against hippocampal neuron apoptosis.

In conclusion, the present study found that hyperglycemia promoted hippocampal neuronal apoptosis by activating the JNK/MLCK signaling pathway, reducing Bcl-2 expression and increasing Bax expression in diabetic rats with cognitive impairment. Green tea reduced the occurrence of hippocampal neuronal apoptosis by inhibiting activation of the JNK/MLCK signaling pathway, increasing Bcl-2 expression and reducing Bax expression and these effects ultimately protected against diabetic cognitive dysfunction.

\section{Acknowledgements}

Not applicable.

\section{Funding}

The present study was supported by the National Natural Science Foundation of China (grant nos. 81960151 and 81960822), the Guizhou Provincial Department of Education Innovation Group Major Research Project [grant no. (2018)021] and the Guizhou Provincial Science and Technology Department Support Program [grant no. (2019)2802].

\section{Availability of data and materials}

The datasets used and/or analyzed during the current study are available from the corresponding author on reasonable request.

\section{Authors' contributions}

WP and XL designed the study, YX and SL and LZ conducted all the experiments. LD, WQ and JZ interpreted and analyzed the data, drafted the manuscript and revised it critically for important intellectual content. All authors read and approved the final manuscript. WP, XL and YX were responsible for confirming the authenticity of the raw data.

\section{Ethics approval and consent to participate}

All animal experiments were approved by the Guizhou Medical University Animal Care and Use Committee (approval no. 1800954; Guiyang, China) and were conducted in accordance with the National Institutes of Health guidelines for the Care and Use of Laboratory Animals.

\section{Patient consent for publication}

Not applicable.

\section{Competing interests}

The authors declare that they have no competing interests. 


\section{References}

1. Dong M, Ren M, Li C, Zhang X, Yang C, Zhao L and Gao H: Analysis of metabolic alterations related to pathogenic process of diabetic encephalopathy rats. Front Cell Neurosci 12: 527, 2019.

2. Díaz-Gerevini GT, Daín A, Pasqualini ME, López CB, Eynard AR and Repossi G: Diabetic encephalopathy: Beneficial effects of supplementation with fatty acids $\omega 3$ and nordihydroguaiaretic acid in a spontaneous diabetes rat model. Lipids Health Dis 18: 43, 2019

3. Liu LJ, Lu XJ, Gao JM, Wang RJ and Cheng GX: Effect of the APP17 peptide on diabetic encephalopathy. J Biol Regul Homeost Agents 33: 251-257, 2019.

4. Kim TW and Park HS: Physical exercise improves cognitive function by enhancing hippocampal neurogenesis and inhibiting apoptosis in male offspring born to obese mother. Behav Brain Res 347: 360-367, 2018

5. Zhu S, Min D, Zeng J, Ju Y, Liu Y and Chen X: Transplantation of stem cells from human exfoliated deciduous teeth decreases cognitive impairment from chronic cerebral ischemia by reducing neuronal apoptosis in rats. Stem Cells Int 2020: 6393075, 2020.

6. Yu N, Huang Y, Jiang Y, Zou L, Liu X, Liu S, Chen F, Luo J and Zhu Y: Ganoderma lucidum triterpenoids (GLTs) reduce neuronal apoptosis via inhibition of ROCK signal pathway in APP/PS1 transgenic Alzheimer's disease mice. Oxid Med Cell Longev 2020: 9894037, 2020.

7. Bhusal A, Rahman MH, Lee IK and Suk K: Role of hippocampal lipocalin-2 in experimental diabetic encephalopathy. Front Endocrinol (Lausanne) 10: 25, 2019.

8. Song Y, Liu P, Li Z, Shi Y, Huang J, Li S, Liu Y, Zhang Z, Wang Y, Zhu W, et al: The effect of myosin light chain kinase on the occurrence and development of intracranial aneurysm. Front Cell Neurosci 12: 416, 2018.

9. Mizui T, Sekino Y, Yamazaki H, Ishizuka Y, Takahashi H, Kojima N, Kojima M and Shirao T: Myosin II ATPase activity mediates the long-term potentiation-induced exodus of stable F-actin bound by drebrin A from dendritic spines. PLoS One 9: e85367, 2014

10. Li L, Wu X, Yue HY, Zhu YC and Xu J: Myosin light chain kinase facilitates endocytosis of synaptic vesicles at hippocampal boutons. J Neurochem 138: 60-73, 2016.

11. Zhang HF, Li TB, Liu B, Lou Z, Zhang JJ, Peng JJ, Zhang XJ, Ma QL, Peng J and Luo XJ: Inhibition of myosin light chain kinase reduces NADPH oxidase-mediated oxidative injury in rat brain following cerebral ischemia/reperfusion. Naunyn Schmiedebergs Arch Pharmacol 388: 953-963, 2015.

12. Kuo CY, Chou TY, Chen CM, Tsai YF, Hwang GY and Hwang TL: Hepatitis B virus X protein disrupts stress fiber formation and triggers apoptosis. Virus Res 175: 20-29, 2013.

13. He Y, Wang F, Chen S, Liu M, Pan W and Li X: The protective effect of radix polygoni multiflori on diabetic encephalopathy via regulating myosin light chain kinase expression. J Diabetes Res 2015: 484721, 2015 .

14. Zhu L, Li C, Du G, Pan M, Liu G, Pan W and Li X: High glucose upregulates myosin light chain kinase to induce microfilament cytoskeleton rearrangement in hippocampal neurons. Mol Med Rep 18: 216-222, 2018.

15. Akchiche N, Bossenmeyer-Pourié C, Pourié G, Koziel V, Nédélec E, Guéant JL and Daval JL: Differentiation and neura integration of hippocampal neuronal progenitors: Signaling pathways sequentially involved. Hippocampus 20: 949-961, 2010

16. Zhao Y, Xin Y and Chu H: MC4R is involved in neuropathic pain by regulating JNK signaling pathway after chronic constriction injury. Front Neurosci 13: 919, 2019.

17. Jiang X, Kannan A and Gangwani L: ZPR1-dependent neurodegeneration is mediated by the JNK signaling pathway. J Exp Neurosci 13: 1179069519867915, 2019.

18. Li Q, Xue AY, Li ZL and Yin Z: Liraglutide promotes apoptosis of HepG2 cells by activating JNK signaling pathway. Eur Rev Med Pharmacol Sci 23: 3520-3526, 2019.

19. Wei H, Ren Z, Tang L, Yao H, Li X, Wang C, Mu C, Shi C and Wang H: JNK signaling pathway regulates the development of ovaries and synthesis of vitellogenin $(\mathrm{Vg})$ in the swimming crab Portunus trituberculatus. Cell Stress Chaperones 25: 441-453, 2020.

20. Suzuki T, Pervin M, Goto S, Isemura $M$ and Nakamura $Y$ : Beneficial effects of tea and the green tea catechin epigallocatechin-3-gallate on obesity. Molecules 21: E1305, 2016.
21. Di Lorenzo A,Nabavi SF, Sureda A, Moghaddam AH, Khanjani S, Arcidiaco P, Nabavi SM and Daglia M: Antidepressive-like effects and antioxidant activity of green tea and GABA green tea in a mouse model of post-stroke depression. Mol Nutr Food Res 60: 566-579, 2016.

22. Pervin M, Unno K, Takagaki A, Isemura M and Nakamura Y: Function of green tea catechins in the Brain: Epigallocatechin gallate and its metabolites. Int J Mol Sci 20: E3630, 2019.

23. Liu S, Liu G, Xu Y, Gao C, Pan W and Li X: Protective effect of green tea on hippocampal neurons in rats under high glucose. Shandong Medicin 58: 35-39, 2018 (In Chinese).

24. Huang Y, Shi T, Luo X, Xiong H, Min F, Chen Y, Nie S and Xie M: Determination of multi-pesticide residues in green tea with a modified QuEChERS protocol coupled to HPLC-MS/MS Food Chem 275: 255-264, 2019.

25. Wu H and Li S: Long non-coding RNA MT1JP exerts anti-cancer effects in breast cancer cells by regulating miR-92-3p. Gen Physiol Biophys 39: 59-67, 2020.

26. Hsu MH, Sheen JM, Chen YC, Yu HR, Tain YL and Huang LT: Rats with prenatal dexamethasone exposure and postnatal high-fat diet exhibited insulin resistance, and spatial learning and memory impairment: Effects of enriched environment. Neuroreport 31: 265-273, 2020.

27. Wang B, Huang J, Li J and Zhong Y: Control of macrophage autophagy by miR-384-5p in the development of diabetic encephalopathy. Am J Transl Res 10: 511-518, 2018.

28. Kakutani S, Watanabe H and Murayama N: Green tea intake and risks for Dementia, Alzheimer's disease, mild cognitive impairment, and cognitive impairment: A systematic review. Nutrients 11: E1165, 2019

29. Zhai Y, Meng X, Ye T, Xie W, Sun G and Sun X: Inhibiting the NLRP3 inflammasome activation with MCC950 ameliorates diabetic encephalopathy in $\mathrm{db} / \mathrm{db}$ mice. Molecules 23: E522, 2018.

30. Jing YH, Zhang L, Gao LP, Qi CC, Lv DD, Song YF, Yin J and Wang DG: Autophagy plays beneficial effect on diabetic encephalopathy in type 2 diabetes: Studies in vivo and in vitro. Neuroendocrinol Lett 38: 27-37, 2017.

31. Wang Z, Huang Y, Cheng Y, Tan Y, Wu F, Wu J, Shi H, Zhang H, $\mathrm{Yu} \mathrm{X}$, Gao H, et al: Endoplasmic reticulum stress-induced neuronal inflammatory response and apoptosis likely plays a key role in the development of diabetic encephalopathy. Oncotarget 7 : 78455-78472, 2016.

32. Wang S, Ren X, Hu X, Zhou L, Zhang C and Zhang M: Cadmium-induced apoptosis through reactive oxygen species-mediated mitochondrial oxidative stress and the JNK signaling pathway in TM3 cells, a model of mouse Leydig cells. Toxicol Appl Pharmacol 368: 37-48, 2019.

33. Wang T, Li X, Fan L, Chen B, Liu J, Tao Y and Wang X: Negative pressure wound therapy promoted wound healing by suppressing inflammation via down-regulating MAPK-JNK signaling pathway in diabetic foot patients. Diabetes Res Clin Pract 150: 81-89, 2019.

34. Liu J, Zhou S, Qian X, Zhang Y and Zhao J: Over-expressed Bax inhibitor 1 (BI-1) inhibits apoptosis of hippocampal neurons via endoplasmic reticulum IRE1-JNK pathway in rats with subarachnoid hemorrhage. Xibao Yu Fenzi Mianyixue Zazhi 33: 1316-1322, 2017 (In Chinese).

35. Cai Y, Jiang W, Zhou AL, Zhou M and Xu L: Effect of oxymatrine on apoptosis of hippocampal neurons by p38/JNK signaling pathway. Zhongguo Zhongyao Zazhi 42: 731-738, 2017 (In Chinese)

36. Kim JM, Park SK, Guo TJ, Kang JY, Ha JS, Lee S, Lee U and Heo HJ: Anti-amnesic effect of Dendropanax morbifera via JNK signaling pathway on cognitive dysfunction in high-fat diet-induced diabetic mice. Behav Brain Res 312: 39-54, 2016.

37. Lou T, Ma J, Xie Y, Yao G, Fan Y, Ma S and Zou X: Nuanxin capsule enhances cardiac function by inhibiting oxidative stress-induced mitochondrial dependent apoptosis through AMPK/JNK signaling pathway. Biomed Pharmacother 135: $111188,2021$.

38. Wang S, He B, Hang W, Wu N, Xia L, Wang X, Zhang Q, Zhou X, Feng Z, Chen Q, et al: Berberine alleviates tau hyperphosphorylation and axonopathy-associated with diabetic encephalopathy via restoring PI3K/Akt/GSK3 $\beta$ pathway. J Alzheimers Dis 65: 1385-1400, 2018.

39. Lei S, Czerwinska E, Czerwinski W, Walsh MP and MacDonald JF: Regulation of NMDA receptor activity by F-actin and myosin light chain kinase. J Neurosci 21: 8464-8472, 2001. 
40. Kim DY and Helfman DM: Loss of MLCK leads to disruption of cell-cell adhesion and invasive behavior of breast epithelial cells via increased expression of EGFR and ERK/JNK signaling. Oncogene 35: 4495-4508, 2016.

41. He Q, Bao L, Zimering J, Zan K, Zhang Z, Shi H, Zu J, Yang X, Hua F, Ye X, et al: The protective role of (-)-epigallocatechin-3-gallate in thrombin-induced neuronal cell apoptosis and JNK-MAPK activation. Neuroreport 26: 416-423, 2015.

42. Xu H, Wu Q, Peng C and Zhou L: Study on the antiviral activity of San Huang Yi Gan Capsule against hepatitis B virus with seropharmacological method. BMC Complement Altern Med 13: 239, 2013.

43. Siu WS, Ko CH, Wong HL, Gao S, Shum WT, Lau CB, Hung LK and Leung PC: Seropharmacological study on osteogenic effects of post-absorption ingredients of an osteoprotective herbal formula. Chin J Integr Med 23: 25-32, 2017.

44. Chu KO, Wang CC, Chu CY, Choy KW, Pang CP and Rogers MS Uptake and distribution of catechins in fetal organs following in utero exposure in rats. Hum Reprod 22: 280-287, 2007.

45. Molino S, Dossena M, Buonocore D, Ferrari F, Venturini L, Ricevuti $\mathrm{G}$ and Verri M: Polyphenols in dementia: From molecular basis to clinical trials. Life Sci 161: 69-77, 2016.
46. Spagnuolo C, Moccia S and Russo GL: Anti-inflammatory effects of flavonoids in neurodegenerative disorders. Eur J Med Chem 153: 105-115, 2018.

47. Ortiz-López L, Márquez-Valadez B, Gómez-Sánchez A, Silva-LuceroMD,Torres-PérezM,Téllez-BallesterosRI,IchwanM, Meraz-Ríos MA, Kempermann G and Ramírez-Rodríguez GB: Green tea compound epigallo-catechin-3-gallate (EGCG) increases neuronal survival in adult hippocampal neurogenesis in vivo and in vitro. Neuroscience 322: 208-220, 2016.

48. Afzal M, Safer AM and Menon M: Green tea polyphenols and their potential role in health and disease. Inflammopharmacology 23: 151-161, 2015.

c) (i) $(-)$ This work is licensed under a Creative Commons c) ${ }_{\text {EY NC NO }}$ Attribution-NonCommercial-NoDerivatives 4.0 International (CC BY-NC-ND 4.0) License. 